\title{
Conservación preventiva y temperatura de superficie en obras pictóricas en exposición
}

\author{
María del Carmen Bellido-Márquez
}

\begin{abstract}
Resumen: Las condiciones medioambientales en que se encuentran las obras de arte son un factor que determina su conservación. Optimizar estos parámetros beneficia la conservación de las mismas. El Centro José Guerrero (Granada) cuenta con una colección permanente (gráfica y pictórica) del pintor José Guerrero, sobre la que se ha realizado el estudio que nos ocupa. El edifico no ofrece una temperatura similar en sus espacios expositivos, ni la temperatura de superficie de los cuadros en exposición es homogénea en toda su extensión, lo que se ha podido comprobar mediante técnicas analíticas de temperatura de superficie, que han reflejado con claridad las condiciones reales de temperatura de las obras, que también resultan ser diferentes a las mediciones térmicas globales del ambiente de las salas, con las consiguientes alteraciones físicas y químicas que este fenómeno puede originar en los materiales de los trabajos artísticos. Debido a ello, este hecho debe ser valorado, con la intención de evitarlo o minimizarlo convenientemente.
\end{abstract}

Palabras clave: Conservación preventiva, temperatura de superficie, condiciones ambientales, arte contemporáneo, museo, exposición, pintura.

\section{Conservação preventiva e temperatura de superfície em obras pictóricas em exposição}

Resumo: As condições ambientais em que se encontram as obras de arte são um factor que determina a sua conservação. Optimizar estes parâmetros beneficia a conservação das mesmas. O Centro José Guerrero (Granada) possui uma coleção permanente (gráfica e pictórica) deste pintor, sobre a qual foi realizado o presente estudo. O edificio não oferece uma temperatura similar em todos os seus espaços expositivos nem a temperatura da superfície das pinturas em exposição é homogénea em toda a sua extensão. Isto foi demonstrado mediante técnicas analíticas de temperatura da superfície que indicaram, com clareza, as condições reais de temperatura nas obras, que para além disso, eram diferentes das medições térmicas globais do ambiente nas salas, com as consequentes alterações físico-químicas que este fenómeno pode originar nos materiais dos trabalhos artísticos. Esta é a razão pela qual este facto deve ser avaliado, com o objectivo de evitar ou minimizar, convenientemente.

Palavras-chave: conservação preventiva, temperatura de superfície, condições ambientais, arte contemporânea, museu, exposição, pintura.

\section{Preventive conservation and surface temperature of exposed paintings}

\begin{abstract}
The environmental conditions surrounding artworks are a determining factor in their conservation. Optimization of such parameters benefits their conservation. The museum Centro José Guerrero (Granada) has a permanent collection of works from this painter (drawings and paintings), upon which this study was performed. The building does not offer a similar temperature throughout its exhibition spaces and the surface temperature of the paintings on display is not regular on their full extension. This has been demonstrated by surface temperature analysis, which clearly reflected the real conditions of artwork temperature, which are also different from the global room temperature measurements. Such fact can originate physical and chemical alterations at the artwork materials. For this reason, this phenomenon should be assessed, in order to avoid or minimize it, conveniently.
\end{abstract}

Keywords: Preventive conservation, surface temperature, environment conditions, contemporary art, museum, exhibition, painting. 


\section{Introducción y objetivos}

El Centro José Guerrero es un organismo de titularidad pública, situado en la ciudad de Granada, que posee una importante colección permanente, formada por obras del artista granadino José Guerrero.

El edificio del Centro presenta planta irregular y muros exteriores de carga construidos en piedra, que están abiertos por series de ventanas arqueadas. El Centro se articula en cuatro niveles arquitectónicos superpuestos. Cada uno de ellos alberga una sala de exposiciones y todas coinciden en la parte central de la construcción, unas sobre otras. Las paredes de las salas están provistas de aislante y revestidas de mortero de construcción. El diseño horizontal de los espacios expositivos se ve favorecido por la disposición de elementos arquitectónicos de comunicación y movimiento (ascensor y varios tramos de escaleras), que generan una distribución secuencial y discursiva en el recorrido de las exposiciones. Así, queda establecido un orden por el que el espacio deviene en un lugar dinámico que guía al visitante y lo inserta en un discurso lineal, acorde con un modelo de museo actual y contemporáneo.

El inmueble cuenta con un sistema de climatización centralizado, formado por un aparato de la marca CIAT (empresa CIATESA), diseñado para controlar la temperatura, la humedad relativa y la pureza del aire, presentando mal funcionamiento, pues con él no se consigue controlar y unificar la temperatura y la humedad en todo el edificio, sino que se advierten diferencias al respecto en sus salas de exposiciones. Esto se ha podido comprobar mediante mediciones puntuales hechas con un termohigrómetro manual. El sistema de control ambiental debería funcionar adecuadamente, según la normativa referente a la conservación de bienes culturales (Baglioni y Losada, 2000) y la opinión de profesionales especialistas (Herráez y Rodríguez, 1989).

El proyecto de iluminación del Centro compatibiliza luz natural (en pasillos, recibidor de entrada, hueco de escalera y sala cuarta de exposiciones) con artificial (pasillos y salas de exposiciones). El sistema de iluminación artificial, está formado por focos direccionales, colocados en el techo de forma equidistante, que originan "baños de pared". Sus lámparas son halógenas, de la marca OSRAM. Se denominan Halolux Ceram Eco. Su potencia es de 100 Watios (W). La vida de estas lámparas se estima en 2.000 horas de uso, manteniendo sus cualidades inalterables. Su flujo luminoso es de 1.800 lumes $(\mathrm{Im})$ de intensidad. La temperatura de color es de $2.900^{\circ}$ Kelvin (K). Su rendimiento de color es del $100 \%$, lo que permite una reproducción de los colores sin distorsión. Además, los focos poseen filtros de rayos ultravioletas.

La distancia media de los focos de iluminación a los paneles expositivos o a los cuadros en exposición se ha obtenido considerando un foco de luz del techo, instalado sobre un observador de $170 \mathrm{~cm}$ de altura, colocado frente al panel o la pintura elegida, donde incide la luz del foco con una inclinación $45^{\circ}$ sobre un punto que está situado a la altura de los ojos del observador. Con estos parámetros la distancia media de los focos de luz a los paneles expositivos o a las pinturas es $210 \mathrm{~cm}$, aproximadamente.

Por otro lado, la colección permanente del Centro está formada por sesenta obras del artista José Guerrero. Cuarenta de ellas están hechas al óleo sobre lienzo y veinte en técnicas mixtas sobre papel (Guerrero, 2000). Las obras se exponen de forma rotativa, variando de ubicación cada dos o tres meses, según la programación museística. 

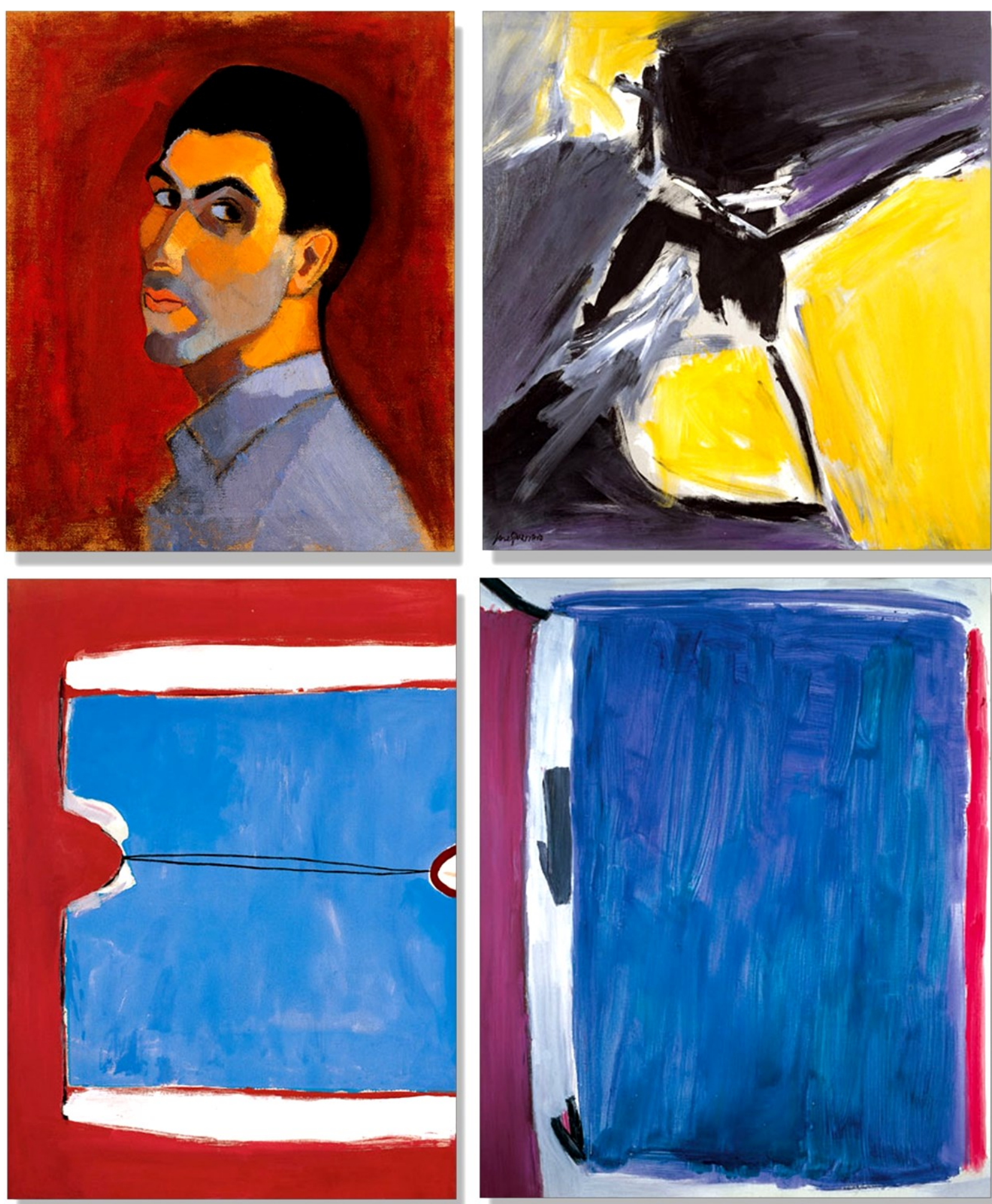

Figura 1: Obras de José Guerrero seleccionadas como material de estudio de esta investigación para realizar los ensayos técnicos de temperatura de superficie. De izquierda a derecha y de arriba abajo se encuentran las obras: Autorretrato (1950), Albacín (1962), Lateral (1974) y Azul añil (1989). @Centro José Guerrero. 
El objetivo general de este estudio es ampliar la información actual sobre la conservación de las obras de arte contemporáneo y aportar datos que ayuden a la resolución de los problemas que plantea (Llamas, 2011), (Herráez y Rodríguez, 1991).

Más concretamente, los objetivos específicos propuestos para este trabajo han sido conocer con exactitud cuáles son las condiciones reales de temperatura de superficie en las obras de la colección durante su exposición en las salas del Centro, y si éstas se ajustan a las recomendaciones de los especialistas en este campo (Guichen y Tapol 1998), comprobando si las temperaturas de superficie de las pinturas se mantienen homogéneas y si difieren o no de las temperaturas ambientales que se registran en las salas. De igual manera, se quiere conocer cuál es la temperatura ambiental media de cada sala y en cuál de ellas el gradiente diferencial de temperatura de superficie de los cuadros es más destacable.

Disponer de los datos indicados supondrá un factor a tener en cuenta en el control de los parámetros medio-ambientales del Centro, lo que ayudará a mejorar los criterios a seguir en la conservación preventiva de las obras (Bellido, 2011), dado que éstas requieren condiciones específicas y adecuadas de conservación preventiva (Calvo, 2002).

\section{Desarrollo metodológico}

Los materiales objeto de este estudio han sido obras pictóricas originales de la colección José Guerrero (Granada) [figura 1], durante su estado de exposición al público. Se han tomado las temperaturas de superficie de las pinturas en exposición y también las temperaturas ambientales de las salas en las que se encontraban expuestas. Cada una de las obras estudiadas ha sido seleccionada por haber estado expuesta en una sala diferente del Centro en el momento de hacer las mediociones del estudio. Todo ello ha supuesto contar con ejemplos prácticos sobre las condiciones térmicas que registran las obras en exhibición en cada sala del edificio.

Todas las mediciones se hicieron sin afluencia alguna de visitantes en el Centro, en días de cierre del museo al público y siempre comenzando a primera hora de la mañana (10:00 h.), estando las obras colgadas de las paredes expositivas de las diferentes salas.

El Termohigrómetro Digital Testo 625 ha sido el instrumento utilizado para la medición de la humedad relativa y la temperatura ambiental de las salas de exposiciones en que se exponían los cuadros a analizar, parámetros que han sido medidos segundos antes de iniciar los registros de las temperaturas de superficie de las obras, en cada ocasión. Este instrumento incluye sonda de humedad integrada, pila y protocolo de calibración. Al encender el termohigrómetro se debe esperar unos segundos antes de realizar mediciones, hasta que comience a funcionar. Sus características principales son: visualización de temperatura y humedad relativa, punto de rocío, valores máximo/mínimo, tecla Hold para retener lecturas, pantalla luminosa y desconexión automática.

El instrumental para obtener las mediciones de temperatura de superficie sobre las obras seleccionadas ha sido un Termómetro de Emisividad Infrarroja Ryatek Inc, Raynger PM 2EM [figura 2]. Este dispositivo es un termómetro digital portátil de superficie sin contacto, que cuenta con puntero láser y detector de rayos infrarrojos. Las características principales de este termómetro son: rango de medición de temperatura de $-18^{\circ}$ a $870^{\circ} \mathrm{C}$, emisividad de 0,10 a 1 (máxima que emite un cuerpo negro), respuesta espectral de 8 a 14 micrones, precisión de $\pm 1 \%$ de lectura de dígito y reproducibilidad de $\pm 0,5 \%$. 


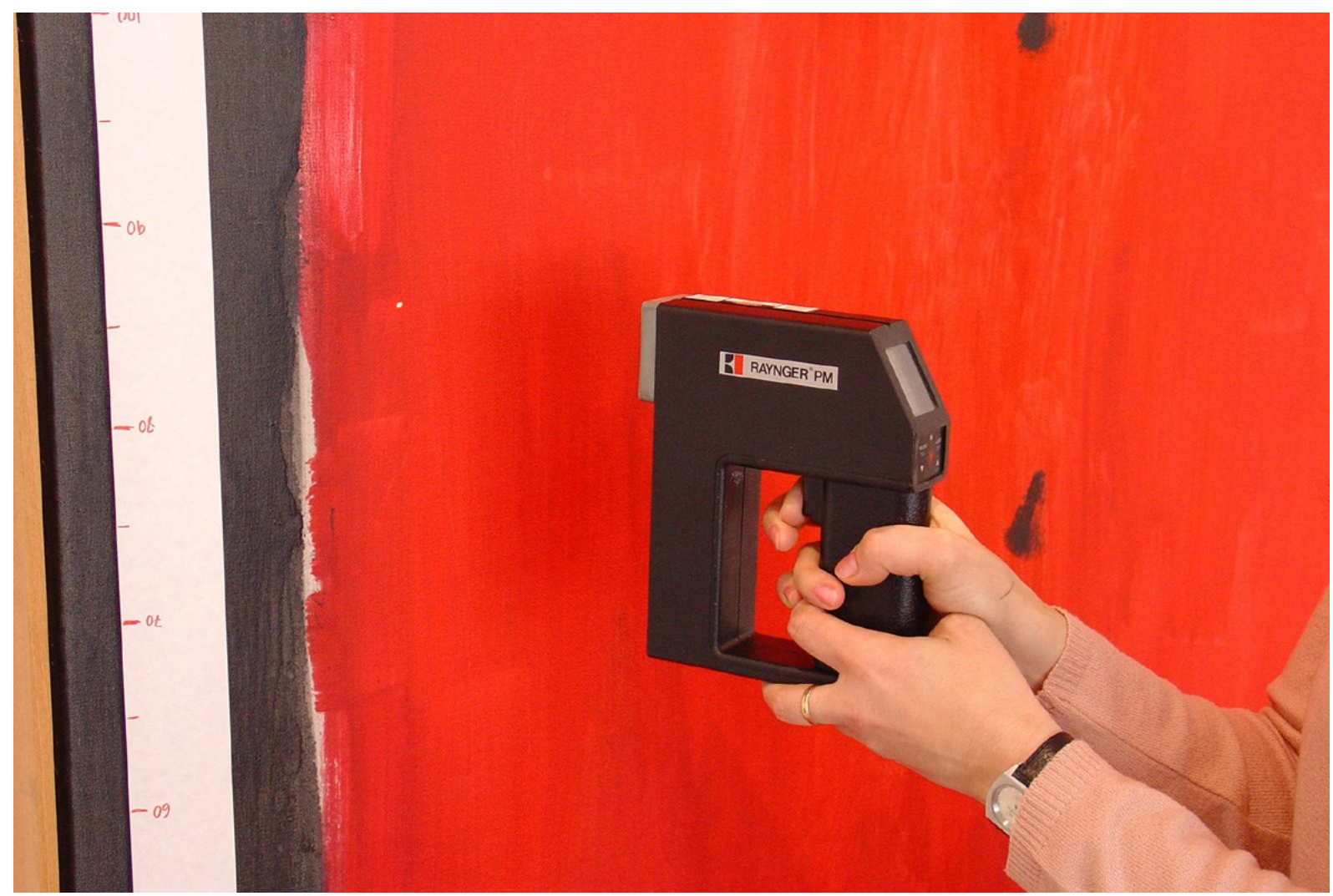

Figura 2: Realización de mediciones de temperatura de superficie sobre una obra de José Guerrero con el Termómetro Portátil Láser de Emisividad Infrarroja Ryatek Inc (Raynger PM 2EM). (María Carmen Bellido-Márquez.

El Termómetro de Emisividad Infrarroja [figura 2] usado permite hacer las mediciones de superficie a distancia, de manera puntual y rápida, registrando la emisividad infrarroja de un punto perteneciente a un cuerpo seleccionado, resultando éstas totalmente inocuas para las obras. EI termómetro tiene un detector de radiación infrarroja que capta la cantidad de energía infrarroja que emite el objeto, la cual se traduce en señal eléctrica y queda expresada numéricamente. El instrumento compensa la cantidad de energía infrarroja captada con la temperatura ambiental, para evitar errores de medición.

Previamente a las mediciones de temperatura de superficie, se han realizado mapas de las obras, para ayudar a definir y localizar cada muestra. Los mapas se han obtenido a partir de imágenes escaneadas de los cuadros a medir. La superficie de cada mapa se ha dividido en cuadrículas de lados regulares y medidas proporcionales a lo que en las obras reales han sido $10 \mathrm{~cm}$. En los puntos de intersección de las líneas se ubicaron las mediciones, para ello se han numerado estos puntos con la intención de denominar y situar las diferentes muestras [figura 3].

Para obtener las mediciones de temperatura sobre la superficie de los cuadros, también se han dividido éstas últimas en cuadrículas regulares de $10 \mathrm{~cm}$ reales de lado y en las intercesiones de las líneas resultantes se han tomado los registros, haciendo coincidir la localización de cada muestra tomada con su referente en el mapa; en él se han anotado los datos de temperatura obtenidos. Así, el trabajo ha resultado organizado y controlado [figura 3,4]. 


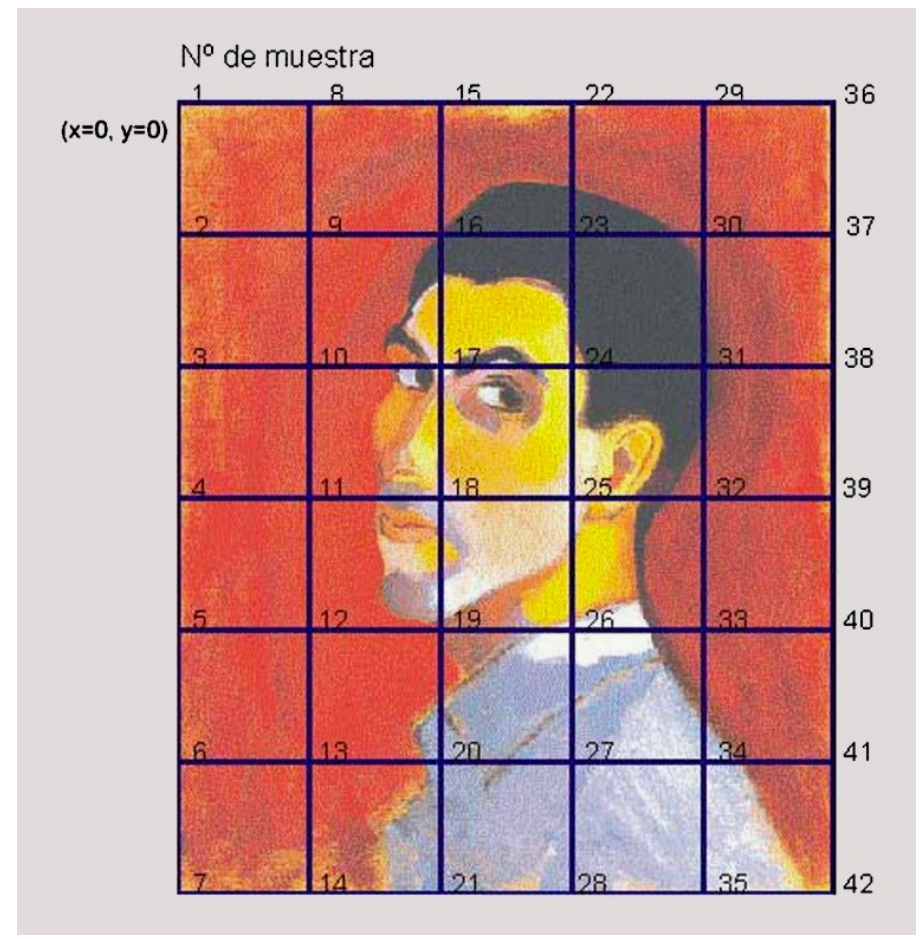

Figura 3: Mapa de trabajo de la obra Autorretrato con la numeración de cada muestra y su localización en la superficie pictórica a medir. OMaría Carmen Bellido-Márquez.

Para dividir las superficies de los cuados se han usado cintas métricas de tela, confeccionadas para este trabajo, con la finalidad de evitar las posibles erosiones que otro tipo de material más rígido o erosivo pudiera provocar en las superficies pictóricas [figura 4].

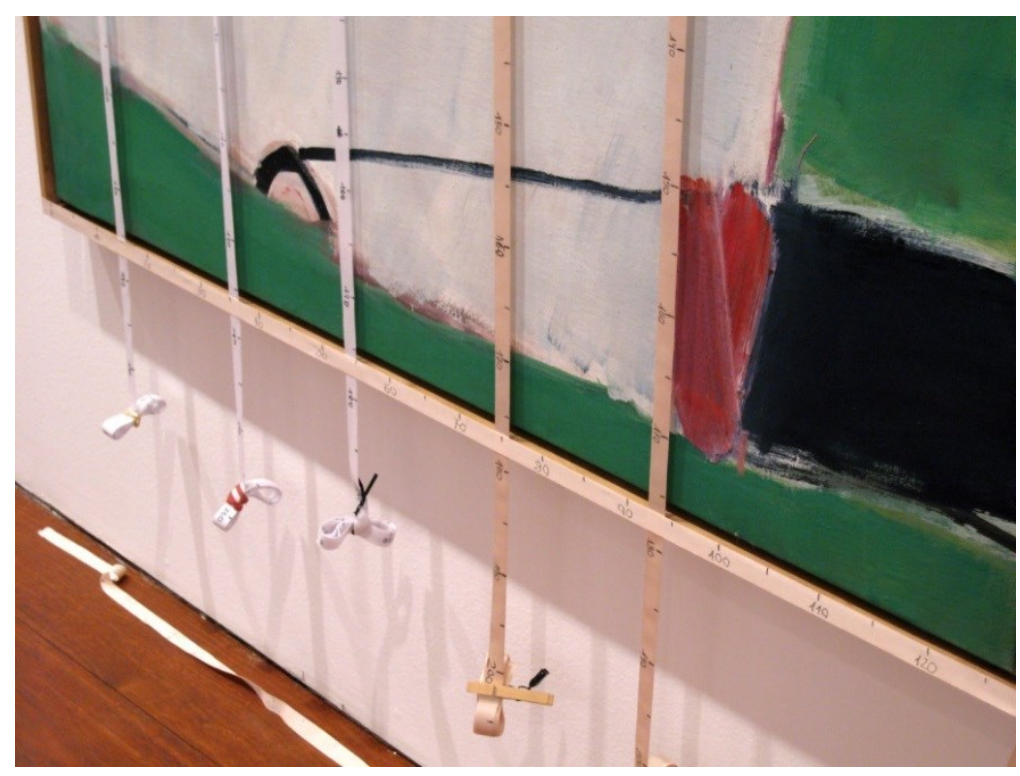

Figura 4: Detalle de la colocación de cintas métricas sobre la obra Litoral durante la toma de muestras, para establecer la localización espacial de los puntos a medir, formando una red superficial cuadriculada. oMaría Carmen Bellido-Márquez. 
Realizadas las mediciones, se han elaborado mapas de isotermas a partir de los datos recogidos. En ello, las temperaturas de superficie se han distribuido en grupos, según escala diferencial de $0,5^{\circ} \mathrm{C}$, y cada grupo de temperaturas ha quedado representado con un color y una letra determinados. De este modo, figuran en los diagramas diversas áreas de colores que corresponden a los diferentes grupos de temperaturas obtenidos, cuyos valores no difieren más de $0,5^{\circ} \mathrm{C}$. entre los muestras de un mismo grupo.

\section{Resultados y discusión}

\section{Autorretrato, 1950. Planta baja.}

La imagen [figura 5] muestra la representación gráfica de los valores procedentes de las mediciones de temperatura de superficie registradas en la obra de José Guerrero Autorretrato (1950). El ensayo se realizó en 2009. La pintura estaba expuesta en la sala primera, planta baja del Centro. Se tomaron 42 datos de temperatura sobre la superficie total del cuadro $(65,5 \times 51 \mathrm{~cm})$. Previamente, se registró la temperatura ambiental $\left(\mathrm{T}^{\mathrm{a}}=22,4^{\circ} \mathrm{C}\right)$ y la humedad relativa $(\mathrm{Hr}=33,9 \%)$ de la sala de exposiciones en que se exponía la obra.
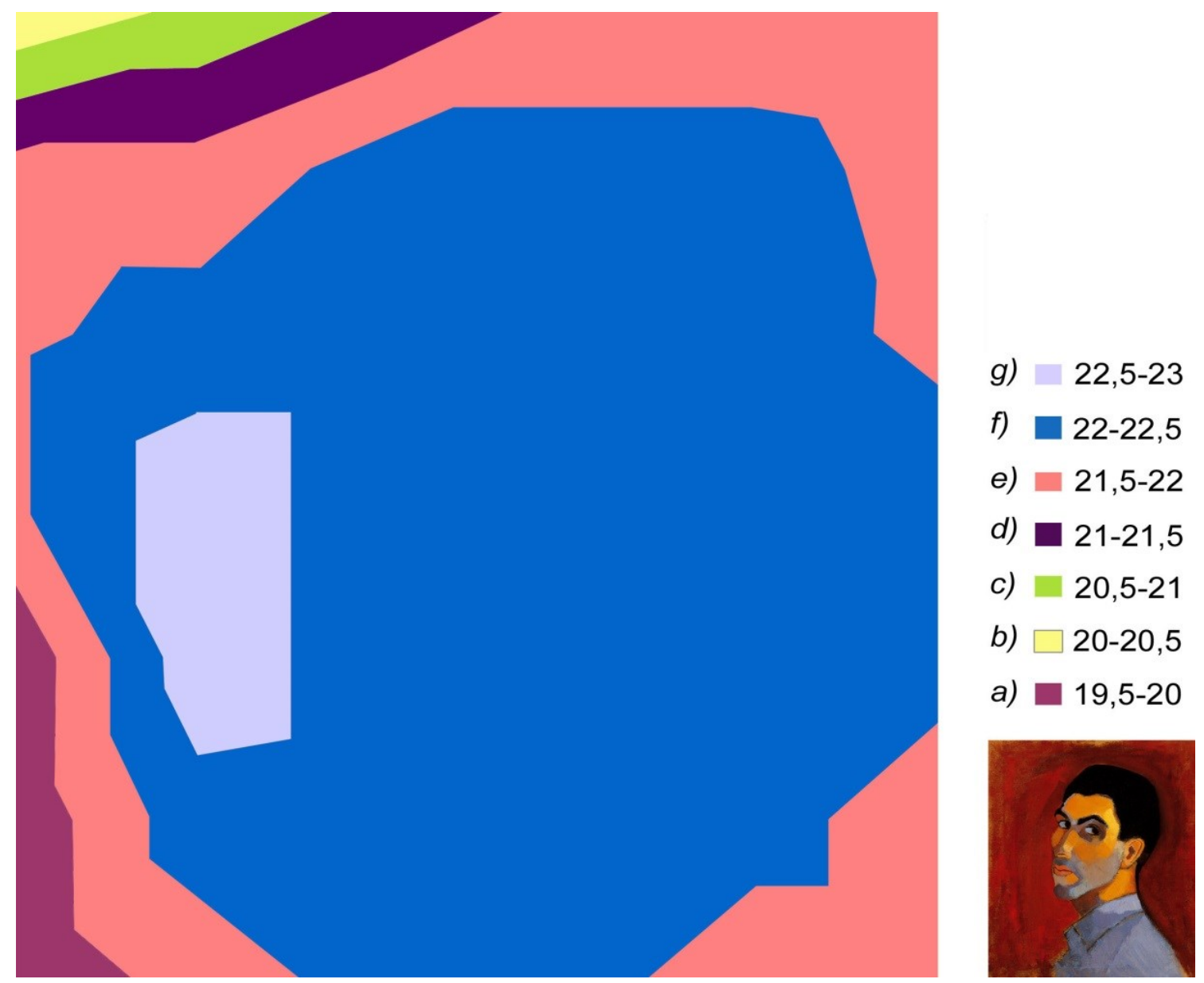

Figura 5: Diagrama de isotermas correspondiente al cuadro Autorretrato, 1950. La escala gráfica (colores y letras) representa variaciones de $0,5^{\circ} \mathrm{C}$. La imagen inferior derecha representa la pintura original. oMaría Carmen Bellido-Márquez. 
En la parte izquierda del gráfico [figura 5] se observan los valores térmicos más variables, que muestran todo el gradiente térmico de la obra. El ángulo inferior izquierdo presenta temperaturas comprendidas entre $19,5^{\circ}-20^{\circ} \mathrm{C}$ (área a, violeta). En el ángulo superior izquierdo de la imagen las temperaturas alcanzan $20^{\circ}-20,5^{\circ} \mathrm{C}$ (área b, beig) y en la mitad izquierda se sitúan las máximas con $22,5^{\circ}-23^{\circ} \mathrm{C}$ (área g, azul celeste). El lado derecho del gráfico muestra valores más uniformes que los anteriores, comprendidos entre $21,5^{\circ} \mathrm{C}$ (área e, salmón) y $22,5^{\circ} \mathrm{C}$ (área f, cian).

Se presenta [figura 5] una distribución térmica en sentido concéntrico, que asciende de fuera hacía dentro. El máximo valor térmico está comprendido entre $22,5^{\circ}-23^{\circ} \mathrm{C}$ (área $\mathrm{g}$, azul celeste). Rodeando el área g, con menor temperatura, está la zona más extensa, formada por el área f (cian), con valores establecidos entre $22^{\circ}-22,5^{\circ} \mathrm{C}$. El área de color salmón (área e) se coloca bordeando la anterior, con valores comprendidos entre $21,5^{\circ}-22^{\circ} \mathrm{C}$, que se encuentran también en los ángulos superior e inferior derechos del diagrama.

Existe una mayor diferencia térmica en la mitad izquierda del gráfico [figura 5] que en la derecha. Las zonas de máxima variabilidad corresponden al cuadrante superior izquierdo, en el que se registran las temperaturas más extremas, frente a las zonas más estables que están en los cuadrantes superior e inferior derechos, con temperaturas muy similares entre sí.

Como resumen, se ha observado en el diagrama de isotermas de la obra Autorretrato [figura 5], situada en la sala de la planta baja, que los valores globales térmicos de superficie del cuadro estuvieron comprendidos entre $19,5^{\circ} \mathrm{C}$ y $23^{\circ} \mathrm{C}$, presentando un gradiente total de $3,5^{\circ} \mathrm{C}$ en toda la superficie, aunque la mayor parte del mismo registró temperaturas establecidas entre $21,5^{\circ} \mathrm{C}$ y $22,5^{\circ} \mathrm{C}$ (áreas e y f), con un gradiente de $1^{\circ} \mathrm{C}$ de diferencia, excepto en su zona lateral izquierda.

\section{Lateral, 1974. Planta $1^{a}$}

La imagen [figura 6] muestra la representación gráfica de los datos obtenidos a partir de las mediciones de la temperatura de superficie, registrados en la obra de José Guerrero Lateral (1974). Se tomaron en 2009 y se realizaron un total de 285 mediciones sobre la superficie total del cuadro $(183 \times 135 \mathrm{~cm})$. La pintura estaba expuesta en la sala segunda del Centro (planta primera). Previamente se registraron la humedad relativa $(\mathrm{Hr}=37,7 \%)$ y la temperatura ambiental $\left(\mathrm{T}^{\mathrm{a}}=24,8^{\circ}\right.$ C) de la sala en que estaba la obra.

En el gráfico [figura 6] la temperatura del cuadrante superior izquierdo presenta valores comprendidos, mayoritariamente, entre $26,5^{\circ}-27,5^{\circ} \mathrm{C}$, representados por las áreas c (beig) y d (cian). En el cuadrante inferior derecho se encuentran temperaturas comprendidas entre $25,5^{\circ}-27^{\circ}$ C en las áreas a (azul celeste), b (violeta) y c (beig).

En el diagrama de isotermas [figura 6] los valores máximos, representados en el área d (cian), se localizan dispersos en varias zonas del cuadrante superior izquierdo, mientras los valores mínimos, representados por el área a (azul celeste), están situados en el cuadrante inferior derecho.

La mayor parte de las zonas [figura 6] muestran temperaturas comprendidas entre $26^{\circ} \mathrm{C}$ y $27^{\circ} \mathrm{C}$, con un gradiente térmico de sólo $1^{\circ} \mathrm{C}$, representadas con las áreas b (violeta) y c (beig). Predomina mayoritariamente el área $\mathrm{C}$, que corresponde a valores entre $26,5^{\circ}-27^{\circ} \mathrm{C}$. 


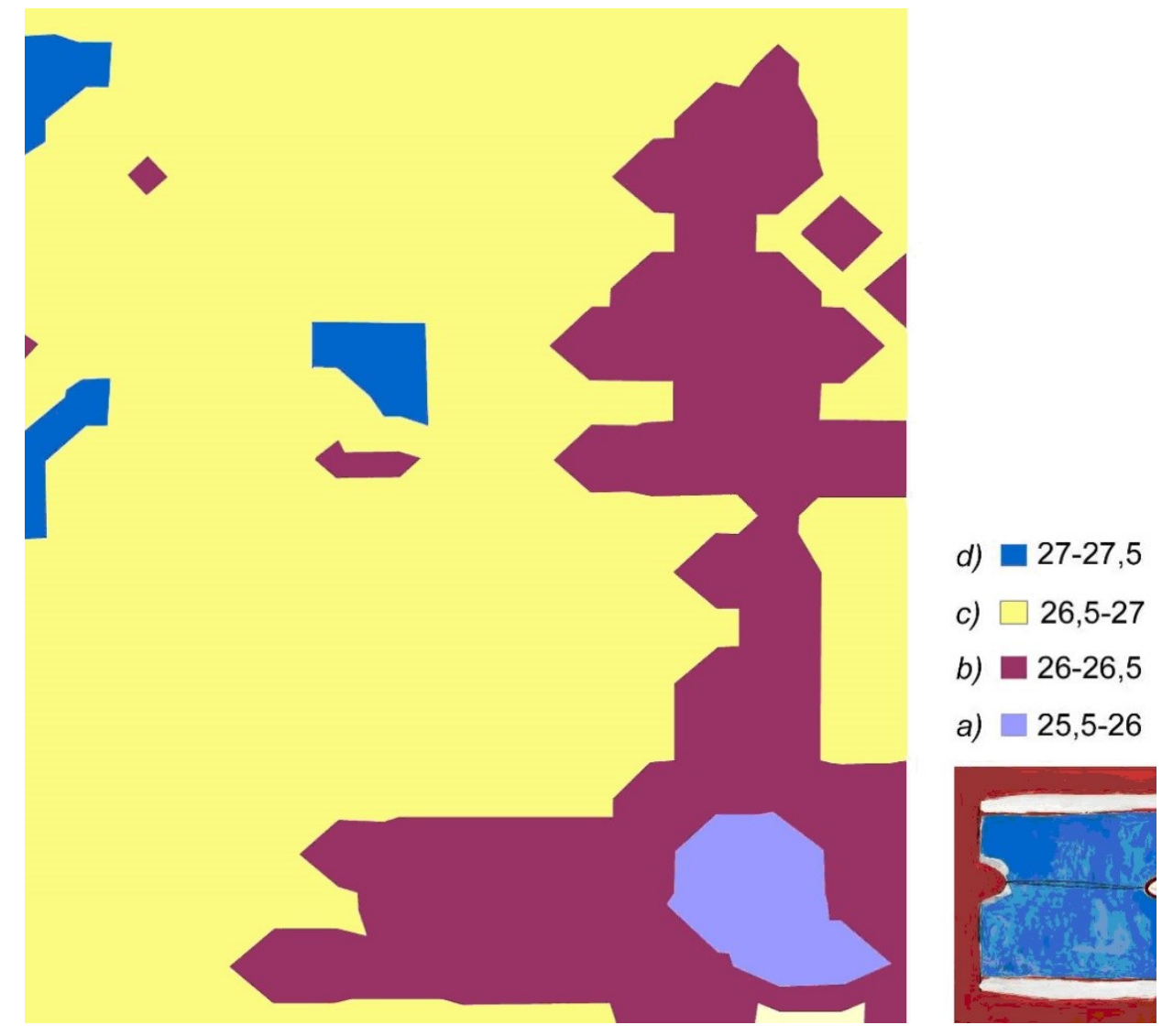

Figura 6: Diagrama de isotermas correspondiente al cuadro Lateral, 1974. La escala gráfica (colores y letras) representa variaciones de $0,5^{\circ} \mathrm{C}$. La imagen inferior derecha representa la pintura original. oMaría Carmen Bellido-Márquez.

El gráfico [figura 6] presenta una variación térmica de temperaturas superiores en el lado izquierdo e inferiores en el derecho. En el primer caso se encuentran, globalmente, valores entre $26^{\circ} \mathrm{C}$ y $27,5^{\circ}$ $\mathrm{C}$, en las áreas b (violeta), $\mathrm{c}$ (beig) y d (cian) y en el segundo las temperaturas oscilan entre $25,5^{\circ} \mathrm{C}$ y $27^{\circ} \mathrm{C}$, estando representadas con las áreas a (azul celeste), b (violeta) y c (beig)).

Resumiendo, el diagrama de isotermas del cuadro Lateral [figura 6], situado en la sala de la planta primera [figura 6] presentó valores térmicos de superficie comprendidos entre $25,5^{\circ} \mathrm{C}$ y $27,5^{\circ} \mathrm{C}$, con un gradiente de $2^{\circ} \mathrm{C}$ en toda la superficie. La temperatura fue descendente de izquierda a derecha de la obra, siendo el descenso más acusado en dirección diagonal, desde el ángulo superior izquierdo al ángulo inferior derecho de la misma.

\section{Albaicín, 1962. Planta 2a}

La imagen [figura 7] muestra la representación gráfica de los valores obtenidos a partir de las mediciones de temperatura de superficie, registradas en la obra de José Guerrero Albaicín (1962). Fueron realizadas en 2009, cuando la pintura estaba expuesta en la sala tercera del Centro (planta segunda). Previamente, se registraron la humedad relativa $(\mathrm{Hr}=38,9 \%)$ y la temperatura ambiental $\left(\mathrm{T}^{\mathrm{a}}=24,4^{\circ} \mathrm{C}\right)$ de la sala. Se tomaron 342 datos de temperatura de superficie, abarcando la totalidad de la extensión de la pintura $(178 \times 168 \mathrm{~cm})$. 


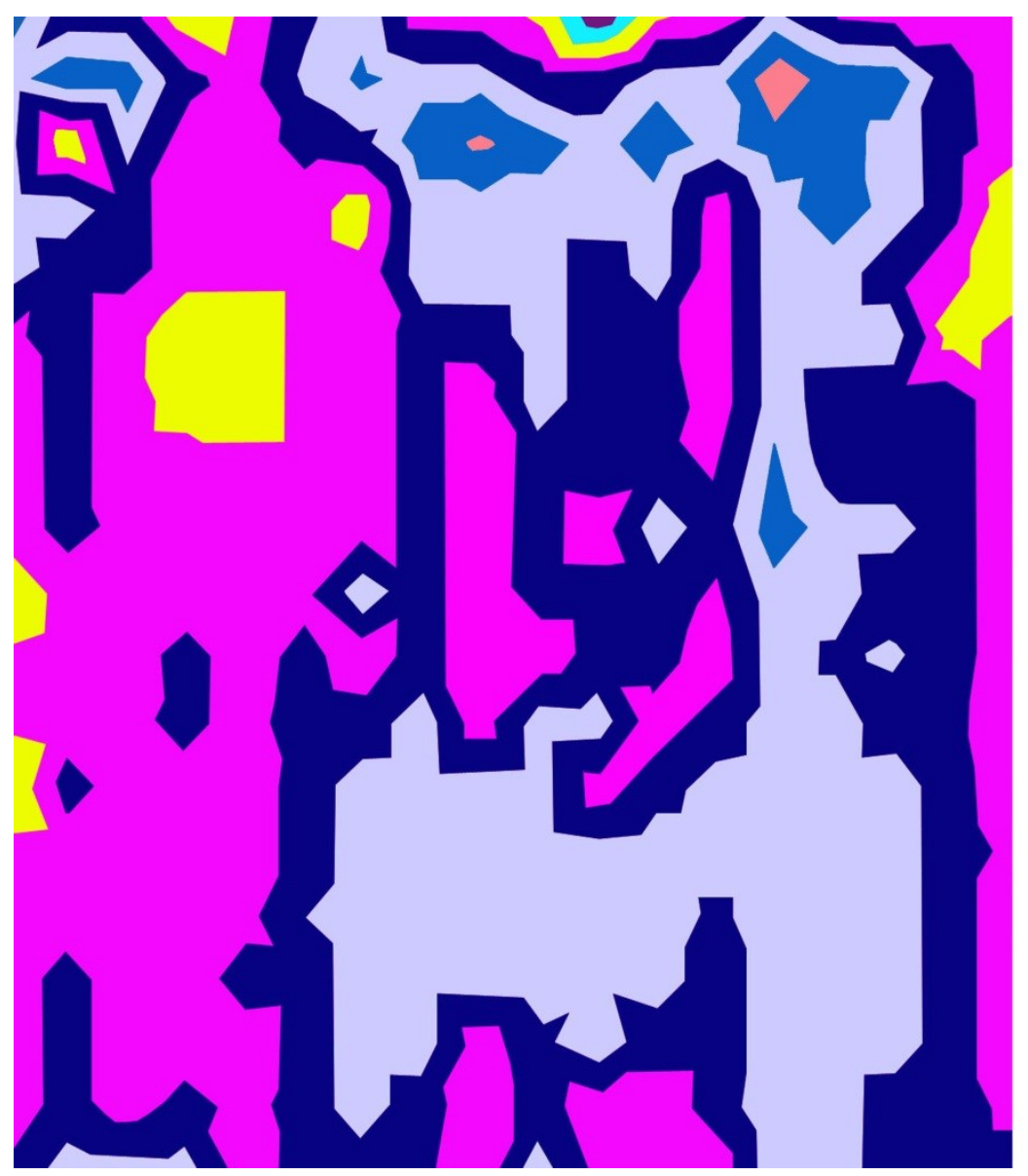
h) $\square 29,5-30$
g) $\square 29-29,5$
f) $\quad 28,5-29$
e) $28-28,5$
d) $27,5-28$
c) $\quad 27-27,5$
b) $\square 26,5-27$
a) $\square 26-26,5$

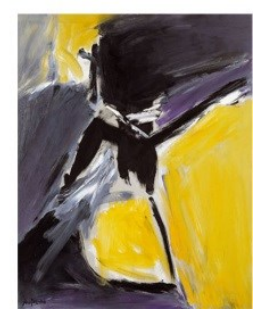

Figura 7: Diagrama de isotermas correspondiente al cuadro Albaicín, 1962. La escala gráfica (colores y letras) representa variaciones de $0,5^{\circ} \mathrm{C}$. La imagen inferior derecha representa la pintura original.

cMaría Carmen Bellido-Márquez.

En la mitad superior del diagrama [figura 7] se encuentran los valores térmicos más elevados, representados por las áreas $\mathrm{f}$ (amarilla), $\mathrm{g}$ (verde) y $\mathrm{h}$ (violeta), que corresponden a temperaturas comprendidas entre $28,5^{\circ} \mathrm{C}$ y $30^{\circ} \mathrm{C}$, siendo las máximas las del área h, (violeta) con $29,5^{\circ}-30^{\circ} \mathrm{C}$, situada en el margen superior del diagrama, con una distribución muy focalizada. En esta misma mitad de la representación se encuentran los registros mínimos $\left(26^{\circ}-27^{\circ} \mathrm{C}\right)$, identificados por las áreas a (salmón) y b (cian). Los más bajos están entre $26^{\circ}-26,5^{\circ} \mathrm{C}$ (área $\mathrm{a}$, salmón), con una ubicación muy cercana a la temperatura máxima. La parte superior del diagrama presenta la mayor variabilidad térmica por zonas, acentuada en el cuadrante superior derecho.

La mitad inferior, el gráfico [figura 7] registra valores térmicos comprendidos entre $27^{\circ} \mathrm{C}$ y $29^{\circ} \mathrm{C}$, con las áreas c (azul celeste), d (azul marino), e (rosa) y f (amarilla). Esta parte es más estable térmicamente.

La focalización de los valores máximos puede obedecer a la irradiación de rayos de iluminación (rayos infrarrojos).

Resumiendo, el gráfico de isotermas del cuadro Albaicín [figura 7], situado en la sala de la segunda (planta segunda) presentó una temperatura de superficie de valores comprendidos entre $26^{\circ} \mathrm{C}$ y 
$30^{\circ} \mathrm{C}$, con un gradiente térmico total de $4^{\circ} \mathrm{C}$. Los valores máximos y mínimos se situaron en la parte superior central y derecha de la superficie pictórica, de forma focalizada, con más incidencia en esta zona de los máximos. La parte inferior de la misma registró mayor estabilidad térmica.

\section{Azul Añil, 1989. Planta 3a}

La imagen [figura 8] muestra la representación gráfica de los valores obtenidos de las mediciones de temperatura de superficie realizadas en la obra de José Guerrero Azul añil (1989), registradas en 2009. Se tomaron un total de 320 mediciones sobre la superficie total del cuadro $(185 \times 145 \mathrm{~cm})$, que estaba expuesto en la sala cuarta el Centro (planta tercera), habiendo medido previamente en ella la temperatura ambiental $\left(\mathrm{T}^{\mathrm{a}}=23,8^{\circ} \mathrm{C}\right)$ y la humedad relativa $(\mathrm{Hr}=34,9 \%)$.

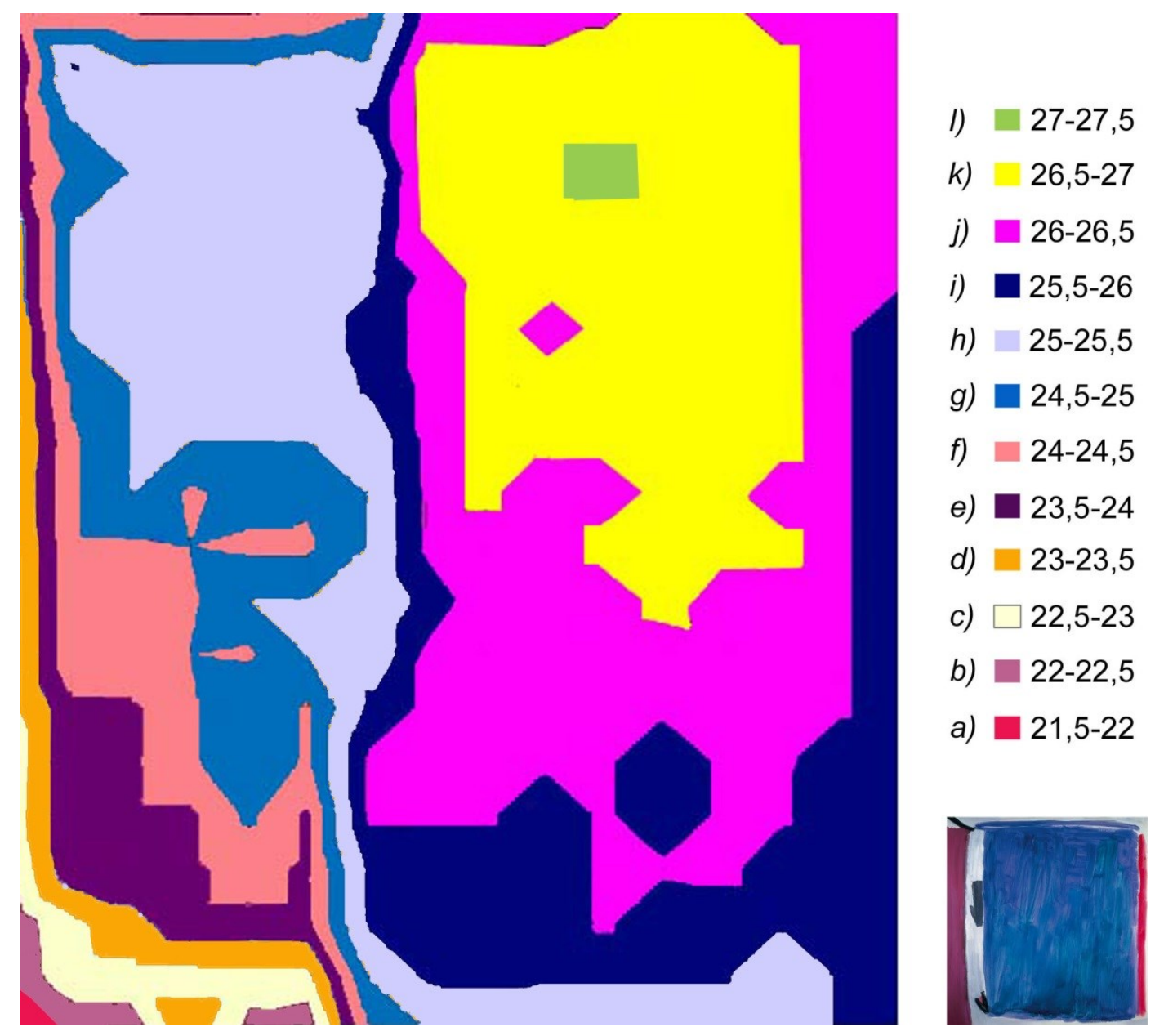

Figura 8: Diagrama de isotermas correspondiente al cuadro Azul Añil, 1989. La escala gráfica (colores y letras) representa variaciones de $0,5^{\circ} \mathrm{C}$. La imagen inferior derecha representa la pintura original. c María Carmen Bellido-Márquez.

En el diagrama [figura 8] se observa que el lado izquierdo de la obra registra valores térmicos comprendidos entre $21,5^{\circ} \mathrm{C}$ y $27^{\circ} \mathrm{C}$, definidos por las áreas: a (rojo), b (lila), c (beig), d (naranja), e (violeta), f (salmón), g (cian), h (azul celeste), i (azul marino), j (rosa) y k (amarilla). El valor térmico 
localizado en el ángulo inferior izquierdo corresponde al mínimo total registrado, con temperaturas comprendidas entre $21,5^{\circ}-22^{\circ} \mathrm{C}$ (área a).

El lado derecho del gráfico [figura 8] muestra valores de temperatura que oscilan entre $25^{\circ} \mathrm{C}$ y $27,5^{\circ} \mathrm{C}$, representados por las áreas: $\mathrm{h}$ (azul celeste), i (azul marino), j (rosa), k (amarillo) y l (verde). Ésta última (área l, verde) muestra las máxima temperaturas registradas, comprendidas entre $27^{\circ}$ $27,5^{\circ} \mathrm{C}$.

Globalmente, el gráfico [figura 8] presenta una variabilidad térmica en la superficie del cuadro de valores inferiores en su lado inferior izquierdo, siendo el cuadrante superior derecho donde están incluidas las máximas. La mayor parte del gráfico presenta valores comprendidos entre $23,5^{\circ} \mathrm{C}$ y $27^{\circ} \mathrm{C}$, representados por las áreas: $\mathrm{d}$ (naranja), e (violeta), $\mathrm{f}$ (salmón), g (cian), h (azul celeste), i (azul marino), $\mathrm{j}$ (rosa) y $\mathrm{k}$ (amarilla), con un gradiente térmico de $3,5^{\circ} \mathrm{C}$.

En resumen, el diagrama de isotermas del cuadro Azul añil [figura 8], situado en la sala de la tercera del Centro (planta cuarta), presentó valores de temperatura de superficie comprendidos entre $21,5^{\circ} \mathrm{C}$ y $27,5^{\circ} \mathrm{C}$, con un gradiente térmico global de $6^{\circ} \mathrm{C}$, ofreciendo los máximos y mínimos de forma focalizada. La variabilidad térmica fue descendente de derecha a izquierda y de arriba debajo de la obra, siendo particularmente acusada en dirección diagonal descendente, desde el cuadrante superior derecho al cuadrante inferior izquierdo de la misma.

\section{Resumen de resultados}

Con los datos recogidos se ha elaborado la siguiente tabla [tabla 1], que permite estudiar comparativamente las variaciones de temperatura registradas en las obras analizadas y relacionarlas con las temperaturas de las salas en las que estaban expuestas durante el estudio.

Tabla 1. Exposición de datos térmicos de temperatura de superficie (máximos, mínimos, gradiente diferencial), temperatura ambiental en sala y ubicación de las obras en el momento de las mediciones. c María Carmen Bellido-Márquez.

\begin{tabular}{|c|c|c|c|c|c|c|c|}
\hline $\begin{array}{l}\text { N.o de } \\
\text { Catalo- } \\
\text { gación }\end{array}$ & $\begin{array}{l}\text { Título } \\
\text { de las } \\
\text { obras }\end{array}$ & $\begin{array}{c}T^{\mathrm{T}} \\
\text { mín. de } \\
\text { superficie }\end{array}$ & $\begin{array}{c}\text { Ta } \\
\text { máx. de } \\
\text { superficie }\end{array}$ & $\begin{array}{l}\text { Gradiente } \\
\text { de Ta de } \\
\text { superficie }\end{array}$ & $\begin{array}{c}\text { Ta } \\
\text { ambiental } \\
\text { de sala }\end{array}$ & $\begin{array}{c}\text { Diferencia de Ta de } \\
\text { sala/Ta máx. de la } \\
\text { obra }\end{array}$ & $\begin{array}{c}\text { Ubicación de las obras } \\
\text { en el Centro } \\
\text { José Guerrero }\end{array}$ \\
\hline 4 & Autorretrato & $19,5^{\circ} \mathrm{C}$ & $23^{\circ} \mathrm{C}$ & $3,5^{\circ} \mathrm{C}$ & $22,4^{\circ} \mathrm{C}$ & $0,6^{\circ} \mathrm{C}$ & Sala planta baja \\
\hline 30 & Lateral & $25,5^{\circ} \mathrm{C}$ & $27,5^{\circ} \mathrm{C}$ & $2^{\circ} \mathrm{C}$ & $24,8^{\circ} \mathrm{C}$ & $2,7^{\circ} \mathrm{C}$ & Sala planta primera \\
\hline 12 & Albaicín & $26^{\circ} \mathrm{C}$ & $30^{\circ} \mathrm{C}$ & $4^{\circ} \mathrm{C}$ & $24,4^{\circ} \mathrm{C}$ & $5,7^{\circ} \mathrm{C}$ & Sala planta segunda \\
\hline 39 & Azul añil & $21,5^{\circ} \mathrm{C}$ & $27,5^{\circ} \mathrm{C}$ & $6^{\circ} \mathrm{C}$ & $23,8^{\circ} \mathrm{C}$ & $3,7^{\circ} \mathrm{C}$ & Sala planta tercera \\
\hline
\end{tabular}

En el cuadro situado en la sala de la planta baja (Autorretrato) el gradiente diferencial de temperaturas de superficie fue de 3,5 y la diferencia entre la máxima temperatura de superficie registrada y la ambiental fue de $0,6^{\circ} \mathrm{C}$.

El cuadro localizado en la sala de la segunda planta (Lateral) fue el que registró menor gradiente diferencial de temperaturas de superficie, con $2^{\circ} \mathrm{C}$ de diferencia total. También presentó $2,7^{\circ} \mathrm{C}$ de diferencia entre la temperatura máxima de superficie y la ambiental. 
El cuadro situado en la sala de la segunda planta (Albaicín) registró un gradiente diferencial de temperatura de superficie de $4^{\circ} \mathrm{C}$ y una diferencia respecto a la ambiental de $5,7^{\circ} \mathrm{C}$, siendo ésta la mayor registrada.

El cuadro ubicado en la sala de la tercera planta (Azul añil) fue el que presentó un gradiente térmico de $6^{\circ} \mathrm{C}$ y también mostró una diferencia de $2,3^{\circ} \mathrm{C}$ entre la temperatura de superficie y la ambiental.

Todos los cuadros registraron temperaturas de superficie variables a lo largo de su extensión superficial. Los gradientes térmicos presentados entre todas las obras quedaron establecidos entre $2^{\circ} \mathrm{C}$ y $6^{\circ} \mathrm{C}$, registrándose los más altos en la obra Azul añil, expuesta en la sala de la planta tercera.

Las diferencias entre temperatura ambiental de sala y la máxima temperatura de superficie de los cuadros fueron de entre $0,6^{\circ} \mathrm{C}$ (Autorretrato, planta baja) y $5,7^{\circ} \mathrm{C}$ (Albacín, planta segunda).

Las temperaturas registradas en las salas estuvieron comprendidas entre los máximos y los mínimos de las registradas en los cuadros correspondientes a ellas.

Los máximos obtenidos de las temperaturas de superficie fueron superiores a las temperaturas ambiental de las salas en todos los casos y también se detectó, puntualmente en algunas mediciones de superficie, que las temperaturas de los cuadros eran inferiores a los de las salas en las que se exponían; ambos casos se deben a que la temperatura de sala es una medida que ofrece una media de ésta, por lo que en su espacio no todas las zonas y superficies han de estar a la temperatura dada.

Teniendo en cuanta que la medida de temperatura recomendada para pintura de caballete debe registrar entre $18^{\circ} \mathrm{C}$ y $20-22^{\circ} \mathrm{C}$, con $\pm 2^{\circ}$ de oscilación (Guichen, Tapol 1988), se observa que los cuadros situados en la primera, segunda y tercera planta exceden la temperatura recomendada.

De los resultados presentados se deduce que los mayores problemas derivados de los parámetros de temperatura de superficie son la falta de homogeneidad térmica dentro de una misma obra y los niveles elevados de temperatura que han presentado las obras y las salas de exposición, todos ellos son superiores a lo recomendado para la adecuada conservación preventiva de las pinturas de José Guerrero, según recomendaciones de Guichen y Tapol (1998) y Calvo (1997).

\section{Conclusiones}

Según se ha comprobado se determina que las condiciones de temperaturas de superficie de los cuadros estudiados no han presentado homogeneidad en toda su extensión y tampoco hay igualdad térmica de superficie entre unas obras y otras, lo que no es apropiado para su adecuada conservación (Rico, 2011).

Cada pintura presentó una máxima de temperatura de superficie superior a la ambiental de la sala en la que se exhibía, de lo que se deduce que sólo el control de la temperatura de la sala no es un dato fiable para conocer la temperatura real de las obras en exposición y que éste resulta insuficiente como único referente térmico de control climático.

La mayor parte de las obras presentaron temperaturas superiores a las recomendadas por los expertos (Guichen, Tapol 1988), especialmente las aquellas situadas en las salas de las plantas segunda, tercera, primera (en orden de mayor a menor incidencia), siendo el exceso y la 
variabilidad climática de la temperatura de superficie la tónica general que presentaron los cuadros.

Como consecuencia de la variabilidad de la temperatura de superficie de los cuadros se concluye determinado que este efecto es debido al sistema de climatización, que no ha funcionado adecuadamente, acondicionando de forma diferente cada sala, y a la iluminación artificial, que ha influido en el calentamiento diferencial de las obras (Casal, 1982). Además, también ha influido en ello el cromatismo de la policromía de los cuadros, dada la absorción diferencial de energía de ciertos puntos de algunas obras. Todos estos factores, combinados conjuntamente, han sido las causas que han afectado a las temperaturas de exposición que han presentado las obras estudiadas. Pero el sistema de climatización es el agente que principalmente ha provocado estas anomalías.

Dado que la temperatura inadecuada es un factor de riesgo para la conservación de las obras de arte (Michalski, 2009), porque favorece el biodeterioro, aumenta la velocidad de reacción de los compuestos químicos y provoca la reducción de humedad, se ha aconsejado realizar las modificaciones de infraestructura necesarias para mejorar las condiciones ambientales de las salas expositivas del Centro José Guerrero, con una prioridad inmediata, mediante el cambio del sistema de climatización. Se indica que el proyecto de mejora ambiental ya se ha realizado con el cierre del Centro por reformas (julio-2013) y se estima que tras la reapertura del Centro en septiembre de 2013 se habrán minimizado los problemas expuestos tras la remodelación del sistema de control ambiental.

\section{Agradecimientos}

Nuestro agradecimiento al Ministerio de Educación y Ciencia por la Beca FPI concedida (2006-2010); a la Universidad Granada por la Beca FPU concedida (2005-2006); al Grupo de Investigación HUM-450 de la Junta de Andalucía (Universidad de Granada), a la familia de José Guerrero, que en todo momento permitió el estudió y la reproducción de imágenes de las obras; al Centro José Guerrero por la colaboración presada; a la Diputación de Granada; y al Departamento de Conservación-Restauración del MNCARS, por colaborar permitiendo el estudio documental de las obras estudiadas.

\section{Bibliografía}

BAGLIONI, R. y LOSADA, J. M. (2000). “Documento de Vantaa (Finlandia). Hacia una estrategia europea sobre conservación preventiva". En Boletín PH, Boletín del Instituto Andaluz del Patrimonio Histórico, no 33, diciembre, pp.87-91.

BELLIDO MÁRQUEZ, M. C. (2012). "Historial conservacional de la obra Variaciones azules. Una pintura contemporánea con problemas de conservación". En Conservación de arte contemporáneo 13a Jornada, febrero 2011. Museo Nacional Centro de Arte Reina Sofía, Departamento de Conservación-Restauración. Ministerio de Cultura, Museo Nacional Centro de Arte Reina Sofía. Págs.129-140. España: Madrid.

CALVO, A. M. (1997). Conservación y restauración: materiales, técnicas y procedimientos: de la A a la Z. Barcelona: Ediciones del Serbal.

CALVO, A. M. (2002). Conservación y restauración de pintura sobre lienzo. Barcelona: Ediciones del Serbal.

CASAL, J. M. (1982). "Alumbrado de museo: bases de su realización". En Museos. Vol. 1: pp. 47-59. Madrid: Patronato Nacional de Museos. 
CENTRO JOSÉ GUERRERO (2012). "La colección del Centro". En Centro José Guerrero. http://www.centroguerrero.org/index.php/del_Centro/19/0/?\&L=0 [Consulta 10 del 1 de 2013].

GUERRERO, J. (2000). Catálogo de la exposición José Guerrero: La colección del Centro. Granada: Diputación de Granada.

GUICHEN, G. DE y TAPOL, B. DE (1998). Climate in museums:measurement. Rome: ICCROM.

HERRÁEZ, J. A. y. RODRÍGUEZ, M. A. (1989). Manual para el uso de aparatos y toma de datos de las condiciones ambientales en museos. Madrid: Dirección General de Bellas Artes y Archivos, D. L.

HERRÁEZ, J. A. y RODRÍGUEZ, M. A. (1991). Recomendaciones para el control de las condiciones ambientales en exposiciones temporales. Madrid: Instituto de Conservación y Restauración de Bienes Culturales.

LLAMAS, R. (Coord.) (2011). Idea, materia y factores discrepantes en la conservación del arte contemporáneo. Valencia: Universidad Politécnica de Valencia.

MICHALSKI S. (2009). Temperatura incorrecta. Canadian Conservation Institute. (Edición en español). http://www.cci-icc.gc.ca/caringfor-prendresoindes/articles/10agents/chap09-spa.pdf [Consulta $10 \mathrm{del} 1 \mathrm{de}$ 2013].

RICO, J. C. (2011). La investigación teórica: museos, del templo al laboratorio: 1986-2011, 25 años de investigación museográfica, el objeto, el sujeto y el espacio. Madrid: Silex.

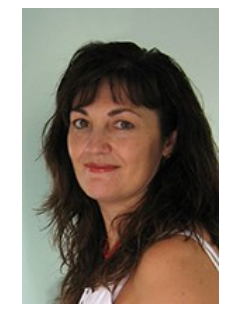

María del Carmen Bellido-Márquez

cbellido@ugr.es

La autora es profesora de Universidad de Granada, adscrita al Departamento de Escultura. Imparte clase en la facultad de Bellas Artes de Granada. Investigador del Grupo de Investigación HUM-951 de la Junta de Andalucía. Posee el Título de Doctorado Europeo en Bellas Artes y Master Propio en Museología por la Universidad de Granada. El tema de su Tesis Doctoral es el estudio de los materiales y la conservación de las obras de arte contemporáneo, centrada en el caso específico de la Colección del Centro José Guerrero (Granada).

Artículo enviado el 31/01/2013

Artículo aceptado el 21/09/2013 